\title{
Frequency of Aortic Arch Variants in Patients with Large Vessel Stroke in the Anterior Circulation
}

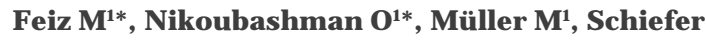
$\mathrm{J}^{2}$, Brockmann $\mathrm{C}^{\mathbf{1}}$, Reich $\mathrm{A}^{\mathbf{2}}$ and Wiesmann $\mathrm{M}^{\mathbf{1}}$

${ }^{1}$ Department of Neuroradiology, RWTH Aachen

University, Germany

${ }^{2}$ Department of Neurology, RWTH Aachen University, Germany

*Corresponding author: Omid Nikoubashman, Department of Neuroradiology, University Hospital, RWTH Aachen University, Pauwelsstr. 30, 52074 Aachen, Germany

Maryam Feiz, Department of Neuroradiology, University Hospital, RWTH Aachen University, Pauwelsstr. 30, 52074 Aachen, Germany

Received: November 21, 2016; Accepted: December 29, 2016; Published: J anuary 02, 2017

\begin{abstract}
Background: The presence of aortic arch variants (AAV) may complicate catheterization and prolong procedure times during endovascular stroke treatment. Hence, it is of great importance to know the prevalence of such variants in stroke patients in order to anticipate possible difficulties during catheterization. As the prevalence of such variants in stroke patients has not been investigated yet, we assessed the prevalence of AAV in patients, who received acute endovascular stroke treatment for large vessel occlusion in the anterior circulation.
\end{abstract}

Methods: We retrospectively analyzed computed tomography angiograms of 248 patients with acute stroke in the anterior circulation, who received endovascular stroke treatment, for the prevalence of a bovine arch, a direct origin of the left vertebral artery, and an aberrant right subclavian artery. We then compared the prevalence of AAV in our stroke cohort with a control cohort of 808 patients.

Results: AAV were found in 152 of all 1056 (14.4\%) patients. There were AAV in 40/248 (16.1\%) patients in the stroke group and 112/808 (13.9\%) patients in the control group $(p=0.374)$. The prevalence of $A A V$ in the stroke and control cohort was as follows: bovine arch: $8.9 \%(22 / 248)$ versus $8.3 \%(67 / 808)$ $(p=0.774)$; direct origin of the left vertebral artery from the aortic arch: $7.3 \%$ $(18 / 248)$ versus $4.6 \%(37 / 808)(p=0.097)$; aberrant right subclavian artery: $0.8 \%$ $(2 / 248)$ versus $1.2 \%(10 / 808)(p=0.742)$.

Conclusion: The prevalence of aortic arch variants in patients with stroke in the anterior circulation is comparable to the prevalence in cohorts without stroke.

Keywords: Stroke; Angiography; Thrombectomy; Aortic arch; CT angiography

\section{Introduction}

Endovascular stroke treatment has become a common treatment technique for acute ischaemic stroke when caused by large vessel occlusion (LVO) [1-5]. Prospective randomized trials showed that endovascular treatment is associated with favorable functional outcome (modified Rankin scale, $\mathrm{mRS} \leq 2$ at day 90 ) in up to $60 \%$ of cases [1]. Khatri, et al. identified rapid recanalization as one of the major keys to favorable functional outcome [6]. It has been shown that endovascular access to the occlusion site can be achieved within minutes via inguinal puncture and catheterization of the aortic arch in the majority of cases [7]. Sometimes, however, catheterization of the aortic arch can be very time-consuming or may even prove to be impossible, hereby affecting clinical outcome [7-13]. Ribo, et al. suggested that the presence of aortic arch variants (AAV) such as the bovine arch might prolong procedure times in endovascular stroke treatment [7]. In order to anticipate possible difficulties during catheterization, it is of great importance to know the prevalence of such variants in stroke patients. Satti, et al. hypothesized that anomalous origins of the arteries of the brain might lead to altered haemodynamics [14]. Hence, it is conceivable that anatomical variants might predispose patients to the formation of atherosclerosis, which might in turn increase the risk for stroke. This would again result in a higher prevalence of AAV in stroke patients. However, to the best of our knowledge, the prevalence of such variants in stroke patients has not been investigated yet. We therefore aimed to compare the prevalence of AAV in patients, who received endovascular treatment for acute ischaemic stroke in the anterior circulation, with a control group of patients without stroke.

\section{Materials and Methods}

\section{Patients}

After obtaining ethical approval from our local ethics board, we retrospectively searched our prospectively maintained stroke registry for all patients with acute ischaemic stroke, who received endovascular stroke treatment between February 2010 and January 2015. We identified 374 patients who fulfilled these criteria. We excluded all 55 patients with stroke in the posterior circulation from our analysis. We also excluded 71 of the remaining 319 patients, for whom no computed tomography angiography (CTA) was available, or in whom CTA data did not allow evaluation of the aortic arch due to incomplete image acquisition or imaging artifacts. This left 248 patients, who received endovascular treatment for acute ischaemic stroke in the anterior circulation, to be included in our study. The
Austin J Cerebrovasc Dis \& Stroke - Volume 4 Issue 1 - 2017 ISSN : 2381-9103 | www.austinpublishinggroup.com Feiz and Nikoubashman et al. (C) All rights are reserved
Citation: Feiz M, Nikoubashman O, Müller M, Schiefer J, Brockmann C, Reich A, et al. Frequency of Aortic Arch Variants in Patients with Large Vessel Stroke in the Anterior Circulation. Austin J Cerebrovasc Dis \& Stroke. 2017; 4(1): 1051 


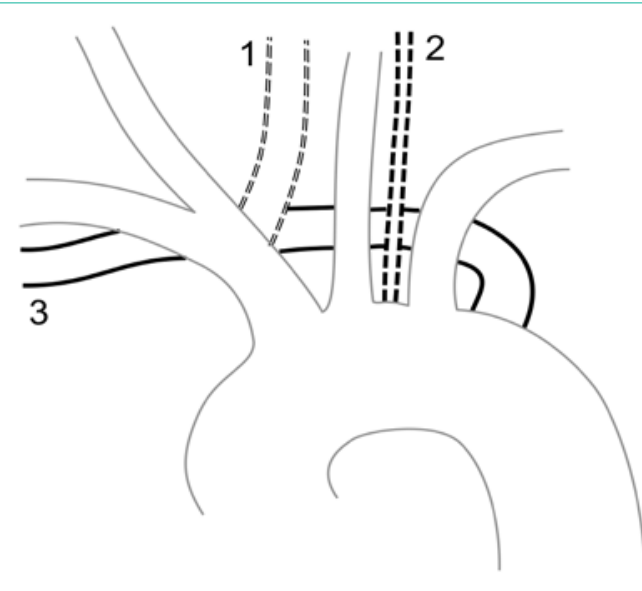

Figure 1: Illustration of Aortic arch variations. 1: Bovine arch with a clea origin of the left common carotid artery from the brachiocephalic artery [17]. 2: direct origin of the left vertebral artery from the aortic arch. 3: aberrant right subclavian artery.

Table 1: Prevalence of aortic arch variations.

\begin{tabular}{|c|c|c|c|c|}
\hline & $\begin{array}{c}\text { Stroke cohort } \\
(\mathrm{n}=248)\end{array}$ & $\begin{array}{c}\text { Control cohort } \\
(\mathrm{n}=808)\end{array}$ & $\begin{array}{c}\text { Total } \\
(\mathrm{n}=1056)\end{array}$ & $\mathbf{p}$ \\
\hline Bovine arch & $22(8.9 \%)$ & $67(8.3 \%)$ & $89(8.4 \%)$ & 0.774 \\
\hline $\begin{array}{c}\text { Direct left vertebral } \\
\text { artery }\end{array}$ & $18(7.3 \%)$ & $37(4.6 \%)$ & $55(5.0 \%)$ & 0.097 \\
\hline $\begin{array}{c}\text { Aberrant right } \\
\text { subclavian artery }\end{array}$ & $2(0.8 \%)$ & $10(1.2 \%)$ & $12(1.1 \%)$ & 0.742 \\
\hline
\end{tabular}

control group was extracted from a previously published dataset [15]. For this aim, we searched for patients without a history of stroke. We identified 808 patients under the age of 60 years, who were all included in this study. We assessed the prevalence of AAV as well as age and sex in all 1056 patients. Stroke aetiology in stroke patients was classified according to the TOAST (Trial of ORG 10172 in Acute Stroke Treatment) classification [16]. Two radiologists, who were blinded to each other's results, analyzed the cohorts independently.

\section{Definitions}

We assessed the prevalence of aortic arch variants, specifically a bovine arch, a direct origin of the left vertebral artery from the aortic arch, and an aberrant right subclavian artery (Figure 1). A bovine arch was defined as the clear origin of the left common carotid artery (CCA) from the brachiocephalic artery, whereas a common trunk of the left CCA and the brachiocephalic artery were not considered as bovine archs [17].

\section{Image acquisition}

CT angiography examinations of the stroke cohort were performed on a 16-slice spiral CT scanner (Siemens SOMATOM Definition AS, Siemens Healthcare, Forchheim, Germany). Arterial contrast in the stroke cohort was achieved by intravenous administration of $80 \mathrm{ml}$ of non-ionic iodinated contrast agent (Iopromid; IsoVist-300) and $30 \mathrm{ml}$ saline solution in an antecubital vein at an injection speed of $5 \mathrm{ml} / \mathrm{sec}$. Technical parameters were as follows: collimation $40 \times 0.6 \mathrm{~mm}$ with reconstruction in $1 \mathrm{~mm}$ slice thickness in an intermediate window; multiplanar reformations in the axial, coronal and sagittal plane (3 $\mathrm{mm}$ slice thickness and $1.5 \mathrm{~mm}$ overlap). Technical parameters of image acquisition of the control cohort were published previously [15].

\section{Statistical analyses}

Pearson's $\mathrm{X}^{2}$ tests were used for comparison of the frequency of AAV in both cohorts. Fisher's exact tests were used when Pearson's $\mathrm{X}^{2}$ tests were not applicable. Age of our cohorts was compared using a Mann-Whitney U test after testing the data for normal distribution with a Shapiro Wilk test. P values under the a level of 0.05 were defined as significant. All statistical analyses were performed with SPSS 23 software (IBM, Armonk, New York).

\section{Results}

Mean age of all patients was 51.516 .7 years (median, 52 years; range, 4 months to 93 years). Overall, 498 (47.2\%) of all included 1056 patients were female. Mean age in the stroke cohort was $71.2 \pm 13.6$ years (median, 74 years; range, 3 to 93 years). There were 138 (55.6\%) female patients. Mean age in the control cohort was $45.5 \pm 12.4$ years (median, 49 years; range, 4 months to 60 years). There were 360 (44.6\%) female patients. As expected the age difference between the two cohorts differed significantly $(\mathrm{p}<0.001)$.

Aortic arch variants were found in 152 of all 1056 (14.4\%) patients. There were AAV in 40/248 (16.1\%) patients in the stroke group and 112/808 (13.9\%) patients in the control group ( $\mathrm{p}=0.374)$. Table 1 and figure 2 illustrate the prevalence and co-occurrence of AAV. Large vessel disease was the only cause of stroke in 53/248 (21.4\%) patients. The prevalence of a bovine arch in these patients $(2 / 53,3.8 \%)$ was nothing her than in patients with other causes of stroke $(20 / 195,10.3 \%)(\mathrm{p}=0.179)$.

\section{Discussion}

Our results are in line with the hypothesis that the prevalence of AAV in stroke patients does not differ from cohorts without stroke. In fact the frequency of AAV in our stroke cohort was not only comparable to our own control cohort but also comparable to the prevalence reported in the literature. Berko, et al. Analyzed CTAs

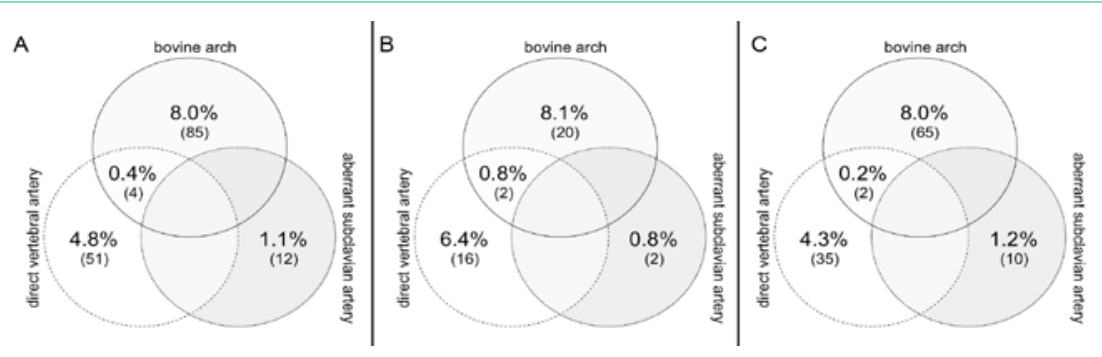

Figure 2: Co-occurrence of aortic arch variants (AAV) in 152 of 1056 (14.4\%) patients with AAV. There were no AAV in 904 of 1056 (85.4\%) patients. A: all patients. $(n=1056)$ B: stroke cohort $(n=248)$. C: control cohort $(n=808)$. 
of 1000 patients with presumed dissections of the aortic arch and reported a prevalence of $7.8 \%$ for a bovine arch, $6.1 \%$ for a direct origin of the left vertebral artery, and $1.2 \%$ for an aberrant right subclavian artery [18]. Faggioli, et al. analyzed 214 patients who underwent carotid artery stenting and reported a similar prevalence of $10.2 \%$ for a bovine arch and $0.9 \%$ for a direct origin of the left vertebral artery. In this context, the results of Jarkani, et al. who analyzed 861 CTAs, stand out. The authors reported a much higher prevalence of $20 \%$ for a bovine arch. However, this high prevalence is likely to be attributed to the authors' differing definition of a bovine arch that also comprised a common trunk of the left CCA and the brachiocephalic artery $[19,20]$. In the end, the frequency of AAV in our stroke cohort was not higher than the prevalence reported in the literature. Also, stroke in the anterior circulation caused by large vessel disease was not more frequent in patients with a bovine arch. Thus, our results disprove the hypothesis that such variants predispose patients to an increased stroke risk in the anterior circulation.

In summary, we have shown that it is not likely that neuro interventionalists encounter aortic arch variant more frequently in patients with stroke in the anterior circulation. Nonetheless, it is conceivable that the presence of AAV might prolong procedural times. Thus, the aim of future studies should be to elucidate whether $\mathrm{AAV}$ are associated with prolonged procedure times.

\section{Limitations}

A major limitation of our study is the fact that our control group was extracted from a previously published cohort that was not matched for age and sex and for whom the exact nature of admission to our hospital could not be specified. Our goal was to compare the prevalence of AAV in patients, who received endovascular treatment for acute ischaemic stroke in the anterior circulation, with a control group of patients without stroke. Despite this limitation our results proved to be highly significant with regard to our own data and with regard to the comparison with the literature.

\section{Conclusion}

The prevalence of $16.1 \%$ of aortic arch variants in patients with stroke in the anterior circulation is comparable to the prevalence in cohorts without stroke. Hence, it is not likely that neuro interventionalists encounter aortic arch variants more frequently in patients with stroke in the anterior circulation.

\section{References}

1. Saver JL, Goyal M, Bonafe A, Diener HC, Levy El, Pereira VM, et al. Stentretriever thrombectomy after intravenous t-PA vs. t-PA alone in stroke. N Engl J Med. 2015; 372: 2285-2295.

2. Jovin TG, Chamorro A, Cobo E, de Miquel MA, Molina CA, Rovira A, et al. Thrombectomy within 8 hours after symptom onset in ischemic stroke. N Engl J Med. 2015; 372: 2296-2306

3. Goyal M, Demchuk AM, Menon BK, Eesa M, Rempel JL, Thornton J, et al Randomized assessment of rapid endovascular treatment of ischemic stroke. N Engl J Med. 2015; 372: 1019-1030.
4. Campbell BC, Mitchell PJ, Kleinig TJ, Dewey HM, Churilov L, Yassi N, et al. Endovascular therapy for ischemic stroke with perfusion-imaging selection. $\mathrm{N}$ Engl J Med. 2015; 372: 1009-1018.

5. Berkhemer OA, Fransen PS, Beumer D, van den Berg LA, Lingsma HF Yoo AJ, et al. A randomized trial of intraarterial treatment for acute ischemic stroke. N Engl J Med. 2015; 372: 11-20.

6. Khatri P, Abruzzo T, Yeatts SD, Nichols C, Broderick JP, Tomsick TA, et al Good clinical outcome after ischemic stroke with successful revascularization is time-dependent. Neurology. 2009; 73:1066-1072.

7. Ribo M, Flores A, Rubiera M, Pagola J, Mendonca N, Rodriguez-Luna D, et al. Difficult catheter access to the occluded vessel during endovascular treatment of acute ischemic stroke is associated with worse clinical outcome. J Neurointerv surg. 2013; 5: i70-i73.

8. Mokin M, Snyder KV, Levy El, Hopkins LN, Siddiqui AH. Direct carotid artery puncture access for endovascular treatment of acute ischemic stroke: technical aspects, advantages, and limitations. J Neurointerv Surg. 2015; 7 : 108-113.

9. Jadhav AP, Ribo M, Grandhi R, Linares G, Aghaebrahim A, Jovin TG, et al. Transcervical access in acute ischemic stroke. J Neurointerv Surg. 2014; 6: 652-657.

10. Wiesmann M, Kalder J, Reich A, Brockmann MA, Othman A, Greiner A, et al. Feasibility of combined surgical and endovascular carotid access for interventional treatment of ischemic stroke. J Neurointerv Surg. 2016; 8: 571575.

11. Faggioli GL, Ferri M, Freyrie A, Gargiulo M, Fratesi F, Rossi C, et al. Aortic arch anomalies are associated with increased risk of neurological events in carotid stent procedures. Eur J Vasc Endovasc Surg. 2007; 33: 436-441.

12. Choi HM, Hobson RW, Goldstein J, Chakhtoura E, Lal BK, Haser PB, et al. Technical challenges in a program of carotid artery stenting. J Vasc Surg. 2004; 40: 746-751.

13. Lin SC, Trocciola SM, Rhee J, Dayal R, Chaer R, Morrissey NJ, et al. Analysis of anatomic factors and age in patients undergoing carotid angioplasty and stenting. Ann Vasc Surg. 2005; 19: 798-804.

14. Satti SR, Cerniglia CA, Koenigsberg RA. Cervical vertebral artery variations: an anatomic study. AJNR Am J Neuroradiol. 2007; 28: 976-980.

15. Müller M, Schmitz BL, Pauls S, Schick M, Röhrer S, Kapapa T, et al. Variations of the aortic arch - a study on the most common branching patterns. Acta Radiol. 2011; 52: 738-742.

16. Adams HP, Bendixen BH, Kappelle LJ, Biller J, Love BB, Gordon DL, et al. Classification of subtype of acute ischemic stroke. Definitions for use in a multicenter clinical trial. TOAST. Trial of Org 10172 in Acute Stroke Treatment. Stroke; a journal of cerebral circulation. 1993; 24: 35-41.

17. Layton KF, Kallmes DF, Cloft HJ, Lindell EP, Cox VS. Bovine aortic arch variant in humans: clarification of a common misnomer. AJNR Am J Neuroradiol. 2006; 27: 1541-1542.

18. Berko NS, Jain VR, Godelman A, Stein EG, Ghosh S, Haramati LB. Variants and anomalies of thoracic vasculature on computed tomographic angiography in adults. J Comput Assist Tomogr. 2009; 33: 523-528.

19. Jakanani GC, Adair W. Frequency of variations in aortic arch anatomy depicted on multidetector CT. Clin Radiol. 2010; 65: 481-487.

20. Layton A, McKay L, Williams D, Garrett V, Gentry R, Sayler G. Development of Bacteroides 16S rRNA gene TaqMan-based real-time PCR assays for estimation of total, human, and bovine fecal pollution in water. Appl Environ Microbiol. 2006; 72: 4214-4224.
Austin J Cerebrovasc Dis \& Stroke - Volume 4 Issue 1 - 2017 ISSN : 2381-9103 | www.austinpublishing group.com

Feiz and Nikoubashman et al. (C) All rights are reserved
Citation: Feiz M, Nikoubashman O, Müller M, Schiefer J, Brockmann C, Reich A, et al. Frequency of Aortic Arch Variants in Patients with Large Vessel Stroke in the Anterior Circulation. Austin J Cerebrovasc Dis \& Stroke. 2017; 4(1): 1051. 